\title{
Relationship of Posterior Decentering of the Humeral Head with Tear Size and Fatty Degeneration in Rotator Cuff Tear
}

\author{
Jung-Han Kim, Hyeong-Won Seo \\ Department of Orthopedic Surgery, Inje University Busan Paik Hospital, Inje University College of Medicine, Busan, Korea
}

\begin{abstract}
Background: Posterior decentering is not an uncommon finding on rotator cuff tear patients' shoulder magnetic resonance imaging. No previous study has reported on the relationship between posterior decentering and rotator cuff tear.

Methods: We assessed patients' rotator cuff tear humeral head positions based on humeral-scapular alignment (HSA). Subjects were classified into centering and decentering groups based on a $<2 \mathrm{~mm}$ or $>2 \mathrm{~mm}$ HSA value, respectively. Differences in rotator cuff tear size, degree of tear, and fatty degeneration between the two groups were evaluated.

Results: One hundred seventy-five patients (80 males, 95 females; mean age: $59.7 \pm 6.5$ years old) were selected as subjects (casecontrol study; level of evidence: 3). Tear size, degree of subscapularis tendon tear, and fatty degeneration of the supraspinatus, infraspinatus, and subscapularis muscles were significantly different between the two groups $(p<0.001, p<0.001, p<0.001)$.

Conclusions: The occurrence of decentering was related to rotator cuff tear size, degree of subscapularis tendon tear, and fatty degeneration of the rotator cuff muscles.
\end{abstract}

(Clin Shoulder Elbow 2019;22(3):121-127)

Key Words: Rotator cuff injuries; Shoulder joint; Decentering; Humeral-scapular alignment

\section{Introduction}

The glenohumeral joint maintains stability through static and dynamic restraints, and the humeral head can be located at the center of the glenoid during the full range of motion of the shoulder joint. The humeral head position on the glenoid in osteoarthritis is an important clinical factor, because it determines the degree of deformation of the glenoid as well as being related to post-arthroplasty prognosis. ${ }^{1-4)}$ There have been many studies on the positional relationship between the glenoid and the humeral head, but most have focused on glenoid wear and retroversion in patients with degenerative arthritis. ${ }^{5-7)}$ Superior migration and anterosuperior escape of the humeral head often is found in massive rotator cuff tears, as well as in cuff tear arthropathy, as a result of altered and unbalanced force couples.
Such migration or escape can affect the choice of treatment options and the clinical outcome after surgery. ${ }^{8-10)}$ Posterior decentering can be found in patients with shoulder instability and dislocation, and it is not an uncommon finding on shoulder magnetic resonance imaging (MRI) in the absence of clinically evident shoulder dislocation..$^{11)}$ However, there are few studies on the factors associated with posterior decentering.

We have observed that the humeral head can be posteriorly displaced to the center of the glenoid in MRI axial images of rotator cuff tear patients who do not have degenerative arthritis or a glenoid deformity or a history of or lesion indicating instability. Thus, we hypothesized that posterior decentering in rotator cuff tear may be related to rotator cuff tendon pathologies or to tear size, tear pattern, or fatty degeneration of associated muscles. The purpose of this study was to evaluate the factors related to

Received March 9, 2019. Revised June 3, 2019. Accepted July 14, 2019.

Correspondence to: Hyeong-Won Seo

Department of Orthopedic Surgery, Inje University Busan Paik Hospital, Inje University College of Medicine, 75 Bokji-ro, Busanjin-gu, Busan 47392, Korea

Tel: +82-51-890-6129, Fax: +82-51-892-6619, E-mail: pointingx77@gmail.com, ORCID: https://orcid.org/0000-0002-4256-1928

IRB approval: Inje University Busan Paik Hospital (No.17-0127).

Financial support: None. Conflict of interests: None. 
posterior decentering in rotator cuff tear patients.

\section{Methods}

Approval for the study was obtained from the Institutional Review Board of Inje University Busan Paik Hospital (No. 170127). Between January 2014 and June 2015, 244 patients underwent surgery for rotator cuff tear and were evaluated for inclusion in this study. All patients underwent surgical treatment only after initial adequate conservative treatment without symptomatic improvement. The subject inclusion criteria were: (1) 50 to 70 years old, (2) had undergone preoperative MRI at our institution within at least 1 month before their operation. The exclusion criteria were: (1) acute traumatic rotator cuff tear, (2) previous history of fractures or surgery on the affected shoulder, (3) arthritic changes of the glenohumeral joint on radiographic studies, (4) history of shoulder dislocation or instability, (5) lesions that might affect stability in MRI (e.g., Bankart lesion or superior labral tear from the anterior to the posterior lesion), (6) presence of posterior synovial hypertrophy, ${ }^{3,2)}$ and (7) internal rotation of humeral head (Fig. 1).

\section{Image Acquisition from MRI}

For indirect magnetic resonance (MR) arthrography, MR contrast material (gadobutrol, Gadavist; Bayer Schering Pharma, Berlin, Germany; $0.1 \mathrm{mmol} / \mathrm{kg}$ body weight) was injected intravenously. Patients were instructed to exercise their shoulders smoothly for 15 minutes, after which MRI were obtained by using a 3 T MRI system (Achieva 3.0 T TX; Philips Medical Systems, Best, Netherlands) with an eight-element phased-array shoulder coil. All scans were acquired with the patient in the supine position, the affected arm by the side, and the hand on the lateral aspect of the thigh. Initially, conventional two-dimensional (2D) MRI was performed, which was followed by threedimensional (3D) isotropic T1-weighted fast-spin-echo (FSE) imaging. Conventional 2D MRI consisted of axial, oblique coronal, and oblique sagittal fat-suppressed T1-weighted FSE sequences and oblique coronal and oblique sagittal T2-weighted FSE sequences. We performed the 3D isotropic, fat-suppressed, T1weighted FSE (volumetric isotropic turbo spin-echo acquisition; Philips Medical Systems) sequence with a $0.5 \mathrm{~mm}$ thickness in the oblique coronal plane. The source data were reformatted into axial and oblique sagittal planes with a $1 \mathrm{~mm}$ thickness. Post-processing was performed by a technologist at the imaging workstation immediately after $\mathrm{MRI}$; the time required for image reformation was approximately 1 minute.

\section{Measurement of Humeral Rotation and Posterior Displacement of the Humeral Head}

Because internal rotation of the humeral head can cause posterior centering, we evaluated the rotation of the humerus but excluded internal rotation groups. Updegrove et al., ${ }^{13)}$ measured humeral rotation by using the following method. First, the axial sequence of an MRI scan was reviewed to determine the most proximal slice showing a definitive outline of the bicipital groove. Then, a circle that best fits the outline of the articular surface of the humeral head was drawn. Two linear lines were drawn through the center of the circle: one along the sagittal plane and the other toward the bicipital groove. The angle between the two lines was measured and recorded as the "humeral rotation". If the bicipital groove was located lateral to the sagittal humeral head centerline, the humerus was designated as "externally rotated" and was assigned a positive angle value. If the bicipital groove was medial to the sagittal humeral head centerline, the

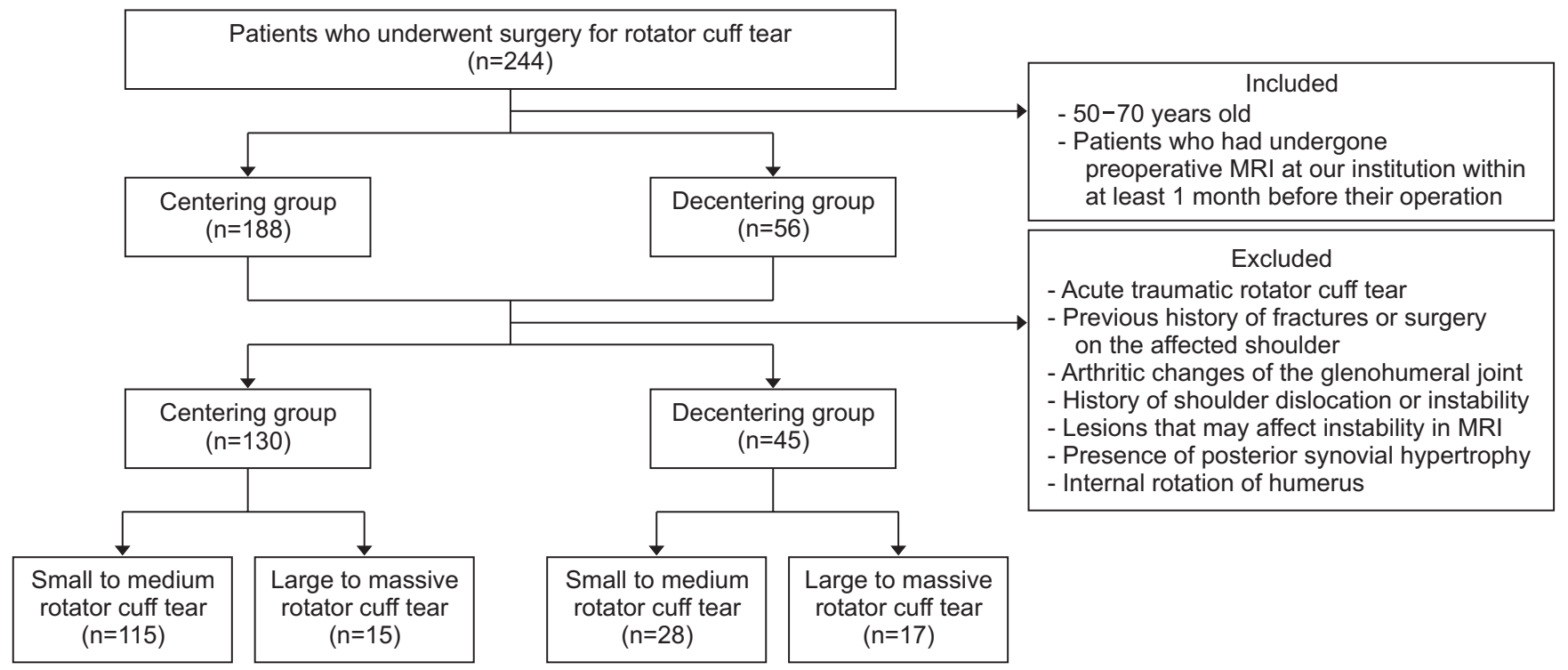

Fig. 1. Flow chart of subject selection and classification. 
humerus was designated as "internally rotated" and was given a negative angle value (Fig. 2). ${ }^{13)}$

A T2-weighted axial view from shoulder MRI was used to measure the humeral-scapular alignment (HSA) at the glenoid center position, with the glenoid center position being confirmed in the sagittal view. To measure the humeral head centering in this image accurately, image measuring software (Rhinoceros 6; McNeel, Seattle, WA, USA) was used. Briefly, the articular surface of the humeral head was assumed to be part of one circle, ${ }^{14)}$ and two points were assigned to the articular surface while one point was assigned to the greater tuberosity area. A circle with the best fit to the humeral head passing these three points was obtained, and after obtaining the best-fit circle, the center of the circle was determined. Next, the HSA, which was the distance between the center of the circle and the line passing through the scapular axis, was determined (Fig. 3). ${ }^{6}$ )

Two orthopedic surgeons, with specialization in the shoulder, took measurements twice at two-week intervals and the results were used to determine interobserver and intraobserver reliability. Based on the position of the humeral head, the patient was classified into either the decentering group or the centering group based on a HAS of $2 \mathrm{~mm}$, as described by Yun et al. $^{15)}$ (centering group: HSA $\leq 2$ mm, 130 patients; decentering group: HSA >2 mm, 45 patients) (Table 1 ).
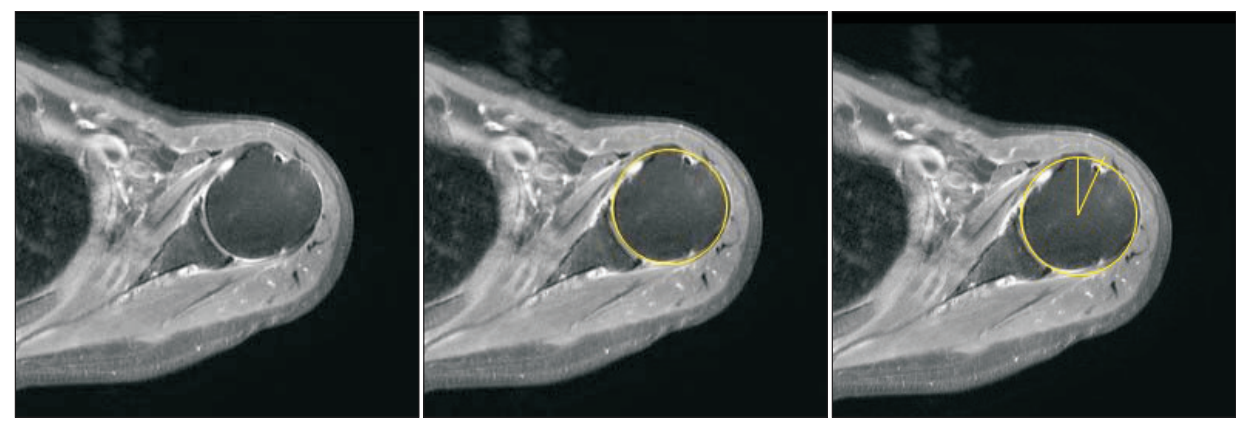

Fig. 2. Measurement of humeral head rotation.

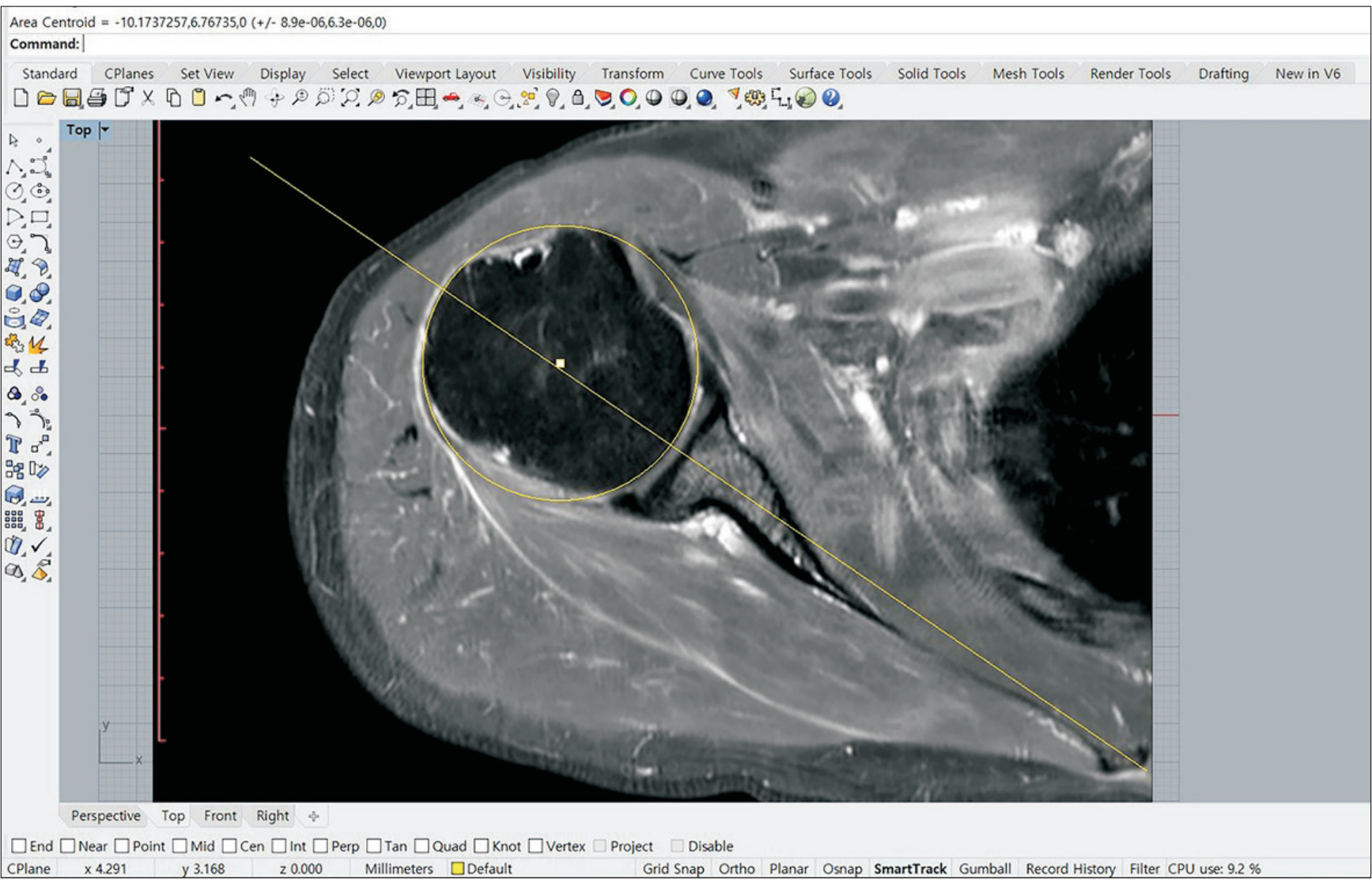

Fig. 3. Measurement of the humeral head center (humeral-scapular alignment [HSA]) by determining the distance between the center of the circle and the line passing through the scapular axis. 
Table 1. Demographic Data of Patient Groups

\begin{tabular}{lccc}
\hline \multicolumn{1}{c}{ Variable } & $\begin{array}{c}\text { Centering } \\
\text { group }\end{array}$ & $\begin{array}{c}\text { Decentering } \\
\text { group }\end{array}$ & p-value \\
\hline Number of patients & 130 & 45 & \\
Dominant arm & $83(63.8)$ & $30(66.7)$ & 0.201 \\
\hline Sex, male/female & $64 / 66$ & $16 / 29$ & 0.235 \\
Age (yr) & $59.6 \pm 6.4$ & $60.9 \pm 7.0$ & 0.300 \\
\hline Symptom duration $(\mathrm{mo})$ & $17.4 \pm 8.1$ & $19.1 \pm 9.2$ & 0.421 \\
\hline Humeral head rotation $\left(^{\circ}\right)$ & $20.2 \pm 5.3$ & $20.8 \pm 8.5$ & 0.581 \\
\hline
\end{tabular}

Values are presented as number only, number (\%), or mean \pm standard deviation.

\section{Tear Characteristics and Fatty Degeneration of Muscles}

From preoperative shoulder MRI, additional data regarding tear size, tear degree, and fatty degeneration of the rotator cuff muscle were collected to evaluate the relationship between decentering and rotator cuff tear. Tear size was evaluated by measuring the mediolateral dimension of the tear; the highest value was selected and the subjects were categorized into four groups: small $(<1 \mathrm{~cm})$, medium $(\geq 1 \mathrm{~cm}$ and $<3 \mathrm{~cm})$, large $(\geq 3 \mathrm{~cm}$ and $<5 \mathrm{~cm}$ ), and massive ( $\geq 5 \mathrm{~cm}$ ). The degree of tear was classified as a full-thickness tear, a partial-thickness tear, or intact. The degree of subscapularis tear was classified using a modification of the Yoo and Rhee classification ${ }^{16}$ : Grade 1: Yoo and Rhee classification 1 and 2a, Grade 2: Yoo and Rhee classification 2b, and Grade 3: Yoo and Rhee classification 3, 4, and 5. In addition, the degree of fatty degeneration of the rotator cuff musculature was examined. Grade 1 indicated some fatty streak, grade 2 indicated fatty streak of no more than $50 \%$ of the muscle, grade 3 indicated fat equal to the muscle, and grade 4 indicated fat content more than $50 \%$ of the muscle. If the degree of fat infiltration varied within the rotator cuff muscle, the highest observed grade was used to assign a grade. ${ }^{17)}$

\section{Statistical Analysis}

Statistical analysis was performed using IBM SPSS ver. 24.0 (IBM Corp., Armonk, NY, USA). The ANOVA was used to analyze the difference in demographic factors between the groups. Student's t-test was used for continuous variables and the Mann-Whitney U-test for non-normal distributions. Categorical variables were tested using the $\chi^{2}$ or Fisher's exact tests. The reliability of the measurements of the radiologic parameters was expressed as an intraclass correlation coefficient (ICC). The ICCs for intraobserver reliability for all measurements (HSA and tear size) were 0.84 and 0.89 , respectively, in MRI. In addition, the HSA and tear size ICCs for interobserver reliability were 0.81 and 0.84 , respectively, in MRI. All test results were assigned a $95 \%$ confidence level. The level of statistical significance was set at $p<0.05$.
Table 2. Comparison of Centering and Decentering Groups of Patients

\begin{tabular}{|c|c|c|c|}
\hline Variable & $\begin{array}{c}\text { Centering } \\
\text { group }(n=130)\end{array}$ & $\begin{array}{l}\text { Decentering } \\
\text { group }(n=45)\end{array}$ & $p$-value \\
\hline Tear dimension (mm) & $16.6 \pm 11.8$ & $25.0 \pm 11.8$ & $<0.001^{\star}$ \\
\hline Tear size ${ }^{\dagger}$ & & & 0.006 \\
\hline Small to medium & $115(88.5)$ & $28(62.2)$ & \\
\hline Large to massive & $15(11.5)$ & $17(37.8)$ & \\
\hline HSA (mm) & $0.73 \pm 0.49$ & $3.84 \pm 2.00$ & $<0.001^{\star}$ \\
\hline \multicolumn{4}{|l|}{ Degree of cuff lesion } \\
\hline Supraspinatus & & & 0.356 \\
\hline FT tear & $91(70.0)$ & $36(80.0)$ & \\
\hline PT tear & $37(28.5)$ & $9(20.0)$ & \\
\hline Intact & $2(1.5)$ & $0(0)$ & \\
\hline Infraspinatus & & & $0.023^{\star}$ \\
\hline FT tear & $19(14.6)$ & $15(33.3)$ & \\
\hline PT tear & $32(24.6)$ & $9(20.0)$ & \\
\hline Intact & $79(60.8)$ & $21(46.7)$ & \\
\hline Subscapularis & & & $0.041^{\star}$ \\
\hline FT tear & 35 (26.9) & $20(44.5)$ & \\
\hline PT tear & $48(36.9)$ & $10(22.2)$ & \\
\hline Intact & $47(36.2)$ & $15(33.3)$ & \\
\hline Yoo and Rhee classification ${ }^{*}$ & & & $<0.001^{\star}$ \\
\hline Grade 1 & $89(68.5)$ & $13(28.9)$ & \\
\hline Grade 2 & $30(23.1)$ & $13(28.9)$ & \\
\hline Grade 3 & $11(8.5)$ & $19(42.2)$ & \\
\hline
\end{tabular}

Fatty degeneration

Supraspinatus

$<0.001^{*}$

Grade 0

$65(50.0)$

$6(13.3)$

Grade 1

37 (28.5)

$16(35.6)$

Grade 2

20 (15.4)

$14(31.1)$

Grade 3

$6(4.6)$

3(6.7)

Grade 4

$2(1.5)$

$6(13.3)$

Infraspinatus

$<0.001^{\star}$

Grade 0

94 (72.3)

19 (42.2)

Grade 1

28 (21.5)

$14(31.1)$

Grade 2

$7(5.4)$

$6(13.3)$

Grade 3
Grade 4

$0(0)$

3(6.7)

Grade 4

$1(0.8)$

3(6.7)

Subscapularis $<0.001^{*}$

\begin{tabular}{lcr} 
Grade 0 & $99(76.2)$ & $13(28.9)$ \\
Grade 1 & $22(16.9)$ & $15(33.3)$ \\
\hline Grade 2 & $6(4.6)$ & $9(20.0)$ \\
\hline
\end{tabular}


Table 2. Continued

\begin{tabular}{|c|c|c|c|}
\hline Variable & $\begin{array}{c}\text { Centering } \\
\text { group }(\mathrm{n}=130)\end{array}$ & $\begin{array}{c}\text { Decentering } \\
\text { group }(n=45)\end{array}$ & $p$-value \\
\hline Grade 3 & $2(1.5)$ & $6(13.3)$ & \\
\hline Grade 4 & $1(0.8)$ & $2(4.4)$ & \\
\hline
\end{tabular}

Values are presented as mean \pm standard deviation or number (\%). HSA: humeral-scapular alignment, FT: full-thickness, PT: partial-thickness. ${ }^{*}$ Indicate statistical significance $(p<0.05) .{ }^{\dagger}$ Evaluated by measuring the mediolateral dimension; small $(<1 \mathrm{~cm})$, medium $(\geq 1 \mathrm{~cm}$ and $<3 \mathrm{~cm})$, large $(\geq 3$ $\mathrm{cm}$ and $<5 \mathrm{~cm})$, and massive $(\geq 5 \mathrm{~cm})$. ${ }^{*}$ The degree of subscapularis tear was classified using a modification of the Yoo and Rhee classification ${ }^{16)}$ (Grade 1: Yoo and Rhee classification 1 and 2a, Grade 2: Yoo and Rhee classification 2b, Grade 3: Yoo and Rhee classification 3, 4, and 5).

\section{Results}

A total of 175 patients (80 males, 95 females; mean age: $59.7 \pm 6.5$ years old), satisfied the inclusion criteria and were selected as study subjects. Among the 175 subjects, decentering was present in 45 cases $(25.7 \%)$ and centering was present in 130 cases $(74.3 \%)$.

\section{Differences in Causes of Decentering in the Two Study Groups}

The average tear size was $25.0 \pm 11.8 \mathrm{~mm}$ in the decentering group and $16.6 \pm 11.8 \mathrm{~mm}$ in the centering group, a significant difference $(p<0.001)$. In the decentering group, there were 28 cases $(62.2 \%)$ of small and medium-sized rotator cuff tears and 17 cases $(37.8 \%)$ of large and massive rotator cuff tears. In the centering group, there were 115 cases (88.5\%) of small and medium-sized rotator cuff tears and 15 cases (11.5\%) of large and massive rotator cuff tears $(p=0.006)$. With regard to the tear degree of each rotator cuff tendon, tears of the supraspinatus were not significantly different between the centering and decentering groups $(p=0.356)$, but the degrees of the infraspinatus and subscapularis tendon tears were significantly different between the two groups ( $p=0.023, p=0.041$, respectively). Based on the modified Yoo and Rhee classification, in the centering group, 89 patients (68.5\%) were grade 1, 30 patients (23.1\%) were grade 2 , and 11 patients ( $8.5 \%$ ) were grade 3 , while in the decentering group, 13 patients (28.9\%) were grade 1, 13 patients (28.9\%) were grade 2, and 19 patients $(42.2 \%)$ were grade 3. There was a significant difference in modified Yoo and Rhee classifications between the two groups $(p<0.001)$. Levels of fatty degeneration of the supraspinatus, infraspinatus, and subscapularis in the centering and decentering groups were significantly different ( $p<0.001, p<0.001, p<0.001$, respectively; Table 2 ). The decentering group had a higher incidence of the high degree of fatty degeneration in each rotator cuff tendon compared to that of the centering group (Table 2).

To concentrate our investigation on differences in patients with smaller tears, we analyzed patients with small to medium rotator cuff tears. Among those patients, the modified Yoo and Rhee classifications of the subscapularis were significantly different between the two groups $(p<0.001)$. In addition, fatty degeneration of the subscapularis was significantly different between the two groups $(p=0.011)$. However, levels of fatty degeneration of the supraspinatus and infraspinatus muscles were not different between the two groups ( $p=0.082, p=0.055$, respectively) (Table 3).

\section{Discussion}

An important finding in the present study was that posterior decentering occurrence increases as the rotator cuff tear size increases and that decentering is also related to the degree of subscapularis tendon tear and to the amount of fatty degeneration of the rotator cuff muscles. Posterior decentering of the humeral head is not uncommon and may be observed in daily clinical practice. Yun et al. ${ }^{15)}$ reported that posterior decentering can present without clinical shoulder instability or a history of shoulder dislocation; in that study accounting for $18.4 \%$ of its prevalence. In the present study, posterior decentering was present in $13.3 \%$ of patients with rotator cuff tear. When rotator cuff tear occurs and the force couples are damaged, translation may occur in the coronal or transverse plane. ${ }^{12)}$ In a biomechanical study, Su et al. ${ }^{9,10)}$ reported that cutting of more than half of the infraspinatus leads to significantly greater posterosuperior translation, and the infraspinatus is a major restraint against posterosuperior translation in rotator cuff tears. In the present study, the mean tear size was $25.0 \pm 11.8 \mathrm{~mm}$ in the decentering group and $16.6 \pm 11.8 \mathrm{~mm}$ in the centering group. Large and massive cuff tears were observed in 17 cases $(37.8 \%)$ in the decentering group, but in only 15 cases (11.5\%) in the centering group. Moreover, fatty degeneration of rotator cuff muscles was significantly different between the two groups; increased levels of fatty degeneration of the supraspinatus, infraspinatus, and subscapularis were present in the decentering group compared to the levels in the centering group $(p<0.001)$. This finding suggests that fatty degeneration, as well as tear size, can be associated with posterior decentering. Yun et al., ${ }^{15}$ in their radiologic evaluation study, also reported that the degrees of fat infiltration of the supraspinatus, infraspinatus, and teres minor muscles observed in the patients' groups with posterior decentering were significantly higher than those in the normal control group.

The subscapularis tendon is important in shoulder stability. Blasier et al. ${ }^{18)}$ reported that the subscapularis provided more resistance to posterior glenohumeral subluxation than that from the supraspinatus and the infraspinatus-teres minor complex. In our study, a significant difference between the decentering and centering groups was observed in the degree of the subscapularis tendon tear and in the amount of fatty degeneration $(p=0.041$, $p<0.001$, respectively). We modified the Yoo and Rhee classifi- 
Table 3. Comparison of Centering and Decentering Group in Small to Medium Rotator Cuff Tear Patients

\begin{tabular}{|c|c|c|c|}
\hline Variable & $\begin{array}{l}\text { Centering group } \\
\quad(\mathrm{n}=115)\end{array}$ & $\begin{array}{c}\text { Decentering } \\
\text { group }(n=28)\end{array}$ & $p$-value \\
\hline Tear size $(\mathrm{mm})$ & $13.3 \pm 7.2$ & $15.4 \pm 8.2$ & 0.184 \\
\hline HSA (mm) & $0.71 \pm 0.46$ & $3.68 \pm 1.91$ & $<0.001^{*}$ \\
\hline \multicolumn{4}{|l|}{ Degree of cuff lesion } \\
\hline Supraspinatus & & & 0.780 \\
\hline FT tear & $77(67.0)$ & $19(67.9)$ & \\
\hline PT tear & $36(31.3)$ & $9(32.1)$ & \\
\hline Intact & $2(1.7)$ & 0 & \\
\hline Infraspinatus & & & 0.307 \\
\hline FT tear & $9(7.8)$ & 0 & \\
\hline PT tear & $29(25.2)$ & $8(28.6)$ & \\
\hline Intact & $77(67.0)$ & $20(71.4)$ & \\
\hline Subscapularis & & & 0.548 \\
\hline FT tear & $24(20.9)$ & $8(28.6)$ & \\
\hline PT tear & $44(38.3)$ & $8(28.6)$ & \\
\hline Intact & $47(40.9)$ & $12(42.8)$ & \\
\hline Yoo and Rhee classification $^{\dagger}$ & & & $<0.001^{*}$ \\
\hline Grade 1 & $85(73.9)$ & $10(35.7)$ & \\
\hline Grade 2 & $22(19.1)$ & $8(28.6)$ & \\
\hline Grade 3 & $8(7.0)$ & $10(35.7)$ & \\
\hline \multicolumn{4}{|l|}{ Fatty degeneration } \\
\hline Supraspinatus & & & 0.082 \\
\hline Grade 0 & $63(54.8)$ & $6(21.4)$ & \\
\hline Grade 1 & $34(29.6)$ & $13(46.4)$ & \\
\hline Grade 2 & $17(14.8)$ & $9(32.2)$ & \\
\hline Grade 3 & $1(0.9)$ & 0 & \\
\hline Grade 4 & 0 & 0 & \\
\hline Infraspinatus & & & 0.055 \\
\hline Grade 0 & $89(77.4)$ & $17(60.7)$ & \\
\hline Grade 1 & $22(19.1)$ & $11(39.3)$ & \\
\hline Grade 2 & $4(3.5)$ & 0 & \\
\hline Grade 3 & 0 & 0 & \\
\hline Grade 4 & 0 & 0 & \\
\hline Subscapularis & & & $0.011^{*}$ \\
\hline Grade 0 & $93(80.9)$ & $10(35.7)$ & \\
\hline Grade 1 & $16(13.9)$ & $10(35.7)$ & \\
\hline Grade 2 & $4(3.5)$ & $4(14.3)$ & \\
\hline Grade 3 & $2(1.7)$ & $4(14.3)$ & \\
\hline Grade 4 & 0 & 0 & \\
\hline
\end{tabular}

Values are presented as mean \pm standard deviation or number (\%). HSA: humeral-scapular alignment, FT: full-thickness, PT: partial-thickness. ${ }^{*}$ Indicate statistical significance $(p<0.05) .{ }^{\dagger}$ The degree of subscapularis tear was classified using a modification of the Yoo and Rhee classification ${ }^{16)}$ (Grade 1: Yoo and Rhee classification 1 and 2a, Grade 2: Yoo and Rhee classification 2b, Grade 3: Yoo and Rhee classification 3, 4, and 5). cation ${ }^{16)}$ for ease of analysis and reclassified the cases into three grades. There was a significant increase in grade level in the decentering group $(p<0.001)$. This result showed that posterior decentering became more frequent with an increase in the subscapularis lesion grade.

Because patients with large and massive cuff tear had a high propensity for concomitant subscapularis tendon tear, we restricted our analysis of tear size by examining only small and medium-sized rotator cuff tears and reanalyzing the differences in subscapularis tendon tear and degree of fatty degeneration between the two groups. Interestingly, even in the small and medium-sized rotator cuff tear patients, decentering was present in $28(19.6 \%)$ of the 143 cases; the degree of subscapularis fatty degeneration was also significantly different between the two groups of patients with small and medium-sized rotator cuff tear $(p=0.011)$. Moreover, with an increase in subscapularis tendon tear, posterior decentering development was more common. ${ }^{15)}$ These results are in line with those in other biomechanical studies that showed that the superior half of the subscapularis significantly alters shoulder biomechanics and leads to increased glenohumeral translation. ${ }^{9,10)}$

This study has some limitations. First, even though we included all patients who were treated within a specific period to correct for selection bias as much as possible, the results are still from a retrospective study of a relatively small patient group. Based on that limitation, a well-designed prospective study with additional randomization is needed. Second, the tear measurement unit was quite small; thus, the results may include measurement error, which might affect the statistical detection of differences when comparing values. To reduce such error, we used a software program that could provide precise measurements.

\section{Conclusion}

Posterior decentering was present in $23.0 \%$ of the patients with a rotator cuff tear and may not be an uncommon finding. Posterior decentering can occur as rotator cuff tear size increases, but it can also develop in patients with small and mediumsized rotator cuff tears. Decentering was related to tear size and to fatty degeneration of the rotator cuff muscles, especially in cases with subscapularis lesions.

\section{References}

1. Hoenecke HR Jr, Tibor LM, D'Lima DD. Glenoid morphology rather than version predicts humeral subluxation: a different perspective on the glenoid in total shoulder arthroplasty. J Shoulder Elbow Surg. 2012;21(9):1136-41. doi: 10.1016/ j.jse.2011.08.044.

2. Hussey MM, Steen BM, Cusick MC, et al. The effects of glenoid wear patterns on patients with osteoarthritis in total 
shoulder arthroplasty: an assessment of outcomes and value. J Shoulder Elbow Surg. 2015;24(5):682-90. doi: 10.1016/j.jse. 2014.09.043.

3. lannotti JP, Norris TR. Influence of preoperative factors on outcome of shoulder arthroplasty for glenohumeral osteoarthritis. J Bone Joint Surg Am. 2003;85(2):251-8. doi: 10.2106/00004623-200302000-00011.

4. Shapiro TA, McGarry MH, Gupta R, Lee YS, Lee TQ. Biomechanical effects of glenoid retroversion in total shoulder arthroplasty. J Shoulder Elbow Surg. 2007;16(3 Suppl):S90-5. doi: 10.1016/j.jse.2006.07.010.

5. Gerber C, Costouros JG, Sukthankar A, Fucentese SF. Static posterior humeral head subluxation and total shoulder arthroplasty. J Shoulder Elbow Surg. 2009;18(4):505-10. doi: 10.1016/j.jse.2009.03.003.

6. Sabesan VJ, Callanan M, Youderian A, lannotti JP. 3D CT assessment of the relationship between humeral head alignment and glenoid retroversion in glenohumeral osteoarthritis. J Bone Joint Surg Am. 2014;96(8):e64. doi: 10.2106/JBJS.L.00856.

7. Terrier A, Ston J, Farron A. Importance of a three-dimensional measure of humeral head subluxation in osteoarthritic shoulders. J Shoulder Elbow Surg. 2015;24(2):295-301. doi: 10.1016/j.jse.2014.05.027.

8. Parsons IM, Apreleva M, Fu FH, Woo SL. The effect of rotator cuff tears on reaction forces at the glenohumeral joint. J Orthop Res. 2002;20(3):439-46. doi: 10.1016/S07360266(01)00137-1.

9. Su WR, Budoff JE, Luo ZP. The effect of posterosuperior rotator cuff tears and biceps loading on glenohumeral translation. Arthroscopy. 2010;26(5):578-86. doi: 10.1016/j.arthro. 2009.09.007.

10. Su WR, Budoff JE, Luo ZP. The effect of anterosuperior rotator cuff tears on glenohumeral translation. Arthroscopy.
2009;25(3):282-9. doi: 10.1016/j.arthro.2008.10.005.

11. King GJ, Richards RR, Zuckerman JD, et al. A standardized method for assessment of elbow function. Research Committee, American Shoulder and Elbow Surgeons. J Shoulder Elbow Surg. 1999;8(4):351-4. doi: 10.1016/S1058-2746(99)901593.

12. Bedi A, Dines J, Warren RF, Dines DM. Massive tears of the rotator cuff. J Bone Joint Surg Am. 2010;92(9):1894-908. doi: 10.2106/JBJS.I.01531.

13. Updegrove GF, Armstrong AD, Mosher TJ, Kim HM. Posterior displacement of supraspinatus central tendon observed on magnetic resonance imaging: a useful preoperative indicator of rotator cuff tear characteristics. Arthroscopy. 2015;31(11):2089-98. doi: 10.1016/j.arthro.2015.04.096.

14. Itoi E, Morrey BF, An K. Biomechanics of the shoulder. In: Rockwood CA Jr, Matsen FA III, Wirth MA, Lippitt SB, eds. The shoulder. 4th ed. Philadelphia: Saunders; 2014. 223-68.

15. Yun G, Kang Y, Ahn JM, et al. Posterior decentering of the humeral head on shoulder MR arthrography: significant association with posterior synovial proliferation. AJR Am J Roentgenol. 2017;208(6):1297-303. doi: 10.2214/AJR.16.17198.

16. Yoo JC, Rhee YG, Shin SJ, et al. Subscapularis tendon tear classification based on 3-dimensional anatomic footprint: a cadaveric and prospective clinical observational study. Arthroscopy. 2015;31(1):19-28. doi: 10.1016/j.arthro.2014.08.015.

17. Goutallier D, Postel JM, Bernageau J, Lavau L, Voisin MC. Fatty muscle degeneration in cuff ruptures. Pre- and postoperative evaluation by CT scan. Clin Orthop Relat Res. 1994;(304):7883.

18. Blasier RB, Soslowsky LJ, Malicky DM, Palmer ML. Posterior glenohumeral subluxation: active and passive stabilization in a biomechanical model. J Bone Joint Surg Am. 1997;79(3):43340 . 